\title{
Exploring non-equilibrium statistical ensembles
}

\author{
BI Qiao* \& LIU Jie \\ Department of Physics, School of Science, Wuhan University of Technology, Wuhan 430070, China
}

Received May 12, 2011; accepted July 7, 2011

\begin{abstract}
Since Gibbs synthesized a general equilibrium statistical ensemble theory, generalizing the Gibbsian theory to non-equilibrium phenomena has been a dream for some theorists. However, the status of the theory for the non-equilibrium ensemble formalism is not as well established as the Gibbsian ensemble theory. In this work, we explore the formalism behind the non-equilibrium statistical ensemble theory based on the sub-dynamic kinetic equation originating from the Brussels-Austin school and developed further by others. The key is establishing a similarity transformation between Gibbsian ensembles and the sub-dynamic ensemble. Using this way, we study a spin-Boson model with strong coupling. A reduced density operator for the canonical ensemble can be easily obtained and the relevant decoherence-free property is revealed.
\end{abstract}

subdynamics, statistical ensemble, non-equilibrium statistical physics

Citation: Bi Q, Liu J. Exploring non-equilibrium statistical ensembles. Chinese Sci Bull, 2011, 56: 3654-3660, doi: 10.1007/s11434-011-4804-5

From the time Gibbs established the equilibrium statistical ensemble theory, many scholars have attempted to generalize the Gibbsian theory to non-equilibrium phenomena domain. However, non-equilibrium statistical ensemble theory cannot be said to be as well established, although important results have been obtained by numerous researchers [1-18]. The literature in this line of research is too numerous to cite here. Instead we just mention three significant developments: the relevant ensembles theory presented by Zubarev et al. [19], the Jaynes' predictive statistical mechanics approach [20], and the generalized Gibbsian ensemble theory based on the Boltzmann kinetic equation presented by Eu [21]. So far the obtained non-equilibrium statistical density distribution formulas for the ensembles do not satisfy the original Liouville equation. Some researchers, for that reason, believe that the Liouville equation needs to have an extra term which satisfies a set of conditions assuring its irreversibility and existence of conservation laws if the Gibbs ensemble theory is to be generalized to non-equilibrium phenomena based on a Liouville-type equation. Since the 1980 s, Xing also proposed a quite interesting non-equi-

*Corresponding author (email: biqiao@gmail.com) librium Liouville equation for classical system and revealed the important role of entropy evolution in non-equilibrium processes [13]. However, finding the extra term for the Liouville equation, which itself possesses universal irreversible characteristic satisfying numerous requirements from numerous models, is still a very difficult open problem. This means that the efforts in establishing a universal ensemble theory covering non-equilibrium phenomena comparable to the Gibbsian ensemble theory is presently still a great challenge.

In this regard, here we present a formalism for non-equilibrium statistical ensemble based on a subdynamic kinetic equation (SKE). SKE evolved from the Brussels-Austin school [22-24] and developed further by others [25,26]. The advantage of the scheme is that SKE intertwines with the original Liouville equation by a similarity transformation without introducing any extra terms in the Liouville equation. If the similarity transformation is non-unitary, the SKE can describe irreversible processes; otherwise, it describes reversible processes as an equivalent equation of the original Liouville equation. Using the established formalism, we discuss the quantum spin-boson model with strong coupling. The reduced density operator for the quantum canonical 
system can be obtained at the level of the master equation approach or beyond. Although there have been several different approaches to construct SKE, which can be found in some publications [22-26], here we try to start from an updated introduction.

\section{Schrödinger type of SKE}

Consider a quantum system (or specifically, some network) $S$ be coupled to a thermal reservoir $B$, and let $H_{S}, H_{B}$, and $H_{\text {int }}$ denote the Hamiltonians of the system $S$, the thermal reservoir $B$, and the interaction between $S$ and $B$, respectively. The total Hamiltonian $H$ of the system plus the reservoir can be expressed as

$$
H_{S} \otimes I_{B}+I_{S} \otimes H_{B}+H_{\text {int }},
$$

and the corresponding quantum Schrödinger equation and Liouville equation are

$$
i \frac{\partial f}{\partial t}=H f
$$

and

$$
i \frac{\partial \rho}{\partial t}=[H, \rho]
$$

where $f$ is the wave function and $\rho$ the density operator for the total system. Then one can introduce a basis, $\left(\phi_{k j}, \varphi_{k j}\right)$, where $j$ is an index denoting $S$ system and $k$ is an index denoting the thermal reservoir $B$. Usually the basis, $\left(\phi_{k j}, \varphi_{k j}\right)$ is chosen as a complete set of eigenvectors of the free Hamiltonian, $H_{S} \otimes I_{B}+I_{S} \otimes H_{B}$; here, in general, the $\left(\left|\phi_{k j}\right\rangle\right.$, $\left.\left\langle\phi_{k j}\right|\right)$ can be chosen as any suitable complete basis in the Hilbert space spanned by the eigenvectors of $H_{S} \otimes I_{B}+$ $I_{S} \otimes H_{B}$. Hence the orthonormal projector, $P_{k j}=\left|\phi_{k j}\right\rangle\left\langle\phi_{k j}\right|$ (or $\left|\varphi_{k j}\right\rangle\left\langle\varphi_{k j}\right| \subseteq Q_{k j}$ ) can be introduced using the basis, with $Q_{k j}=1-P_{k j}$, so that the total Hamiltonian $H=H_{S}+H_{B}+H_{\text {int }}$ can be expressed as

$$
H=P_{k j} H P_{k j}+P_{k j} H Q_{k j}+Q_{k j} H P_{k j}+Q_{k j} H Q_{k j},
$$

giving the eigenvalue problem for the projected matrix of the total Hamiltonian, $\left(\begin{array}{ll}P_{k j} H P_{k j} & P_{k j} H Q_{k j} \\ Q_{k j} H P_{k j} & Q_{k j} H Q_{k j}\end{array}\right)$, described as

$$
\begin{gathered}
P_{k j} H P_{k j} \phi_{k j}+P_{k j} H Q_{k j} \varphi_{k j}=E_{k j} \phi_{k j}, \\
Q_{k j} H P_{k j} \phi_{k j}+Q_{k j} H Q_{k j} \varphi_{k j}=E_{k j} \varphi_{k j},
\end{gathered}
$$

which allows one to solve $\varphi_{k j}$ and $\phi_{k j}$ respectively by

$$
\varphi_{k j}=\left(E_{k j}-Q_{k j} H Q_{k j}\right)^{-1} Q_{k j} H P_{k j} \phi_{k j}=C_{k j} \phi_{k j},
$$

$$
\phi_{k j}=\left(E_{k j}-P_{k j} H P_{k j}\right)^{-1} P_{k j} H Q_{k j} \varphi_{k j}=C_{k j}^{\prime} \varphi_{k j},
$$

Substituting eq. (6) into eq. (4) and eq. (7) into eq. (5) respectively gives

$$
\left(P_{k j} H P_{k j}+P_{k j} H C_{k j}\right) \phi_{k j}=\Theta_{k j} \phi_{k j}=E_{k j} \phi_{k j},
$$

$$
\left(Q_{k j} H C_{k j}^{\prime}+Q_{k j} H Q_{k j}\right) \varphi_{k j}=\Theta_{k j}^{\prime} \varphi_{k j}=E_{k j} \varphi_{k j},
$$

where introducing a creation (annihilation) correlation operator (as a type of resolvent) as

$$
\begin{gathered}
C_{k j}=Q_{k j} C_{k j} P_{k j}=\left(E_{k j}-Q_{k j} H Q_{k j}\right)^{-1} Q_{k j} H P_{k j}, \\
C_{k j}^{\prime}=P_{k j} C_{k j}^{\prime} Q_{k j}=\left(E_{k j}-P_{k j} H P_{k j}\right)^{-1} P_{k j} H Q_{k j} .
\end{gathered}
$$

This shows that the $\left(\phi_{k j}, \varphi_{k j}\right)$ is an eigenvector of the $\left(\Theta_{k j}, \Theta_{k j}^{\prime}\right)$, and $E_{k j}$ is a joint eigenvalue of $\left(\Theta_{k j}, \Theta_{k j}^{\prime}, H\right)$. This allows one to presume that the eigenvector of $H$ is given by $f_{k j}$ with the same eigenvalue $E_{k j}$ by

$$
\begin{aligned}
& P_{k j} f_{k j}=\phi_{k j}=C_{k j}^{\prime} \varphi_{k j}=C_{k j}^{\prime} Q_{k j} f_{k j}=C_{k j}^{\prime} f_{k j}, \\
& Q_{k j} f_{k j}=\varphi_{k j}=C_{k j} \phi_{k j}=C_{k j} P_{k j} f_{k j}=C_{k j} f_{k j} .
\end{aligned}
$$

Hence the eigenvectors of $H$ are related to the eigenvectors of $\Theta$ by eq. (12) plus eq. (13) and considering $P_{k j}^{2}=P_{k j}$,

$$
f_{k j}=\left(P_{k j}+C_{k j}\right) \phi_{k j}
$$

Using eq. (13), by introducing $\Pi_{k j}=\left|f_{k j}\right\rangle\left\langle f_{k j}\right|$ as an eigenprojector of $H$, one can construct a Schrödinger-type of SKE for each projected state $P_{k j} \Pi_{k j}|\phi\rangle$ as $i \frac{\partial}{\partial t} P_{k j} \Pi_{k j}|\phi\rangle=$ $P_{k j} H\left(P_{k j}+C_{k j}\right) \Pi_{k j}|\phi\rangle$, where, for more generality, $\Pi_{k j}$ can be understood as $\left|f_{k j}\right\rangle\left\langle\tilde{f}_{k j}\right|$ in which $f_{k j} \in \Phi$ (dense subspace) and $\tilde{f}_{k j} \in \Phi^{\times}$(generalized dual subspace of $\Phi$ ) are defined in a Rigged Hilbert space, $\Phi \subset H \subset \Phi^{\times}$. Therefore, a Schrödinger-type of SKE can be generally established as

$$
\begin{aligned}
& i \frac{\partial}{\partial t} \phi_{\text {proj }}=\Theta \phi_{\text {proj }}, \\
& i \frac{\partial}{\partial t} \tilde{\phi}_{\text {proj }}=\Theta \tilde{\phi}_{\text {proj }},
\end{aligned}
$$

with

$$
\Theta=\sum_{k j} P_{k j} H\left(P_{k j}+C_{k j}\right)
$$

where $\phi_{\text {proj }}$ and $\tilde{\phi}_{\text {proj }}$ are defined as 


$$
\begin{aligned}
\left|\phi_{\text {proj }}\right\rangle & =\sum_{k j} P_{k j} \Pi_{k j}|\phi\rangle, \\
\left\langle\tilde{\phi}_{\text {proj }}\right| & =\sum_{k j}\langle\tilde{\phi}| P_{k j} \Pi_{k j},
\end{aligned}
$$

and $\phi$ or $\tilde{\phi}$ is a solution of the original Schrödinger equation in the Rigged Hilbert space. We would like to emphasize the above established Schrödinger-type of SKE (SSKE) is different from the old Liouville-type of SKE (LSKE) developed by the Brussels school many years ago. SSKE is important because it is a bridge to the building of non-equilibrium statistical ensemble theory. However, from the times of the Brussels school, researchers always worked on the LSKE and forgot about finding an SSKE. Therefore, it has been difficult to establish a non-equilibrium statistical ensemble theory since that theory needs the eigenvalues of the $\Theta$ operator which intertwine with the Hamiltonian but not with Liouvillian. Of course, based on the SSKE, by replacing $\phi_{\text {proj }}$ and $\tilde{\phi}_{\text {proj }}$ with $\rho_{\text {proj }}=\left|\phi_{\text {proj }}\right\rangle\left\langle\tilde{\phi}_{\text {proj }}\right|$, LSKE can also easily be derived.

The construction of the SSKE (LSKE) in a subspace can be related to the original Schrödinger (Liouville) equation with the same spectral structure between $\Theta$ operator and Hamiltonian (Liouvillian) [22,25]. For instance, using the relation (14) one has the spectral representation of $H$ related to $\Theta$ as $H=\Omega \Theta \Omega^{-1}$, where $\Omega=\sum_{k j}\left(P_{k j}+C_{k j}\right)$. The creation operator, $C_{v}=\frac{1}{Z-Q_{v} H Q_{v}} Q_{v} H P_{v}$, creates the $Q_{v}$-part of $\Pi_{v}$ from the $P_{v}$-part. While $\Theta=H_{0}+\lambda H_{1} C$ is called collision operator [25]. The second-order approximation of $\Theta$ with respect to $\lambda$ corresponds to the master equation [24] derived by the Zwanzig projection technique, and the Boltzmann, Pauli, and Fokker-Planck equations of kinetic theory, and Brownian motion can also be derived by some approximation of $\Theta$ [27]. The author would like to clarify that although using the Zwanzig projection technique, the (differential integral) master equation for the relevant part of the density operator in Liouvillian space can also be derived [26,28], but the Schrödinger type of differential equation (15) in the subspace cannot be derived by the Zwanzig projection technique [29]. Sub-dynamics is more general. Moreover, the formalism can introduce a kind of information holography between the original Schrödinger (Liouville) equation and SKE, which means for every basic dynamical equation one can construct its SKE by a projection procedure, and both are intertwined by similarity transformation (this is also true for the Schwinger-Tomonaga equation and the Einstein equation [26]). Therefore, we make a bold statement here that the universe is holographic through these basic equations, which may be described with the following picture, in which the first index means 1-order of the SKE, the second index means 2-order of the SKE, and so on up to the $n$-order:

$$
\text { Schrodinger (Liouville) eqs. }\left\{\begin{array}{l}
\operatorname{SKE}_{1}\left\{\begin{array}{l}
\operatorname{SKE}_{11} \cdots \\
\operatorname{SKE}_{12} \\
\vdots
\end{array}\right. \\
\operatorname{SKE}_{2}\left\{\begin{array}{l}
\operatorname{SKE}_{21} \cdots \\
\operatorname{SKE}_{22} \cdots \\
\vdots
\end{array}\right. \\
\operatorname{SKE}_{n}\left\{\begin{array}{l}
\operatorname{SKE}_{n 1} \cdots \\
\operatorname{SKE}_{n 2} \cdots \\
\vdots \\
\vdots
\end{array}\right.
\end{array}\right.
$$

Fortunately, this holographic formalism can be used to solve the eigenvalue problem for the Schrödinger (Liouville) equation quite generally as follows: if $P_{k j}=\left|\phi_{k j}\right\rangle\left\langle\phi_{k j}\right|$ is an eigenprojector of $H_{0}$, then from the SKE one has the eigenvector of $H$ given by $\left\langle f_{k j}\right|=\left\langle\phi_{k j}\right|\left(P_{k j}+C_{k j}\right)$, and its eigenvalue given by

$$
\begin{aligned}
E_{k j}= & \left\langle\phi_{k j}\left|H_{0}\right| \phi_{k j}\right\rangle \\
& +\sum_{k j} \frac{1}{E_{k j}-E_{k j}^{q}}\left\langle\phi_{k j}\left|H_{\mathrm{int}} Q_{k j}\right| \phi_{k j}^{q}\right\rangle\left\langle\phi_{k j}^{q}\left|Q_{k j} H_{\mathrm{int}}\right| \phi_{k j}\right\rangle,
\end{aligned}
$$

where introducing $Q_{k j} H Q_{k j}$ and supposing the spectral decomposition of $\frac{1}{E_{k j}-H_{q}}$ is given, the eigenvalue $E_{k j}^{q}$ can be obtained by the SKE again. Continuing this procedure until finally one has $Q_{k j}^{\prime \prime \prime} H Q_{k j}^{\prime \prime \prime \prime}$ only containing one projector, then one can get its eigenvectors and eigenvalues. Replacing the final result into the previous current formalism, one can eventually obtain the eigenvector corresponding to the eigenvalue of $H$.

\section{Subdynamic statistical ensembles}

One notable observation is that the SSKE (or LSKE) seems to have a general property to approach various kinetic equations or master equations that is beyond the original Liouville equation. Furthermore, the LSKE can intertwine with the original Liouville equation using a similarity operator. If this operator is unitary, then, as an equivalent unitary representation of Liouville equation, the Liouville type of SKE is reversible; if the operator is non-unitary, the Liouville type of SKE is irreversible and the corresponding evolution is not time symmetric. This means that the LSKE can be an 
appropriate kinetic equation to describe the irreversible process, in which the evolution operator is non-unitarily realized on generalized functional space which is beyond the traditional Liouville space. This provides motivation to propose using the $\Theta$ operator to construct a non-equilibrium statistical ensemble theory. The construction may be quite simple using the similarity transformation between the Gibbsian ensemble formalism based on the Liouville equation and the non-equilibrium ensemble formalism based on the LSKE (and SSKE): if given the expectation value of the Hamiltonian, then the corresponding expectation of the $\Theta$ operator should be

$$
\operatorname{Tr}(H \rho)=\langle H\rangle \rightarrow \operatorname{Tr}(\Theta P \Pi \rho)=\langle\Theta\rangle .
$$

This allows one to present (by extension) a new canonical ensemble distribution $\rho\left(\theta_{k}\right)$ which is the "vacuum" associated with the "dynamic part" of the original $\rho\left(E_{k}\right)$, as expressed in Balescu's textbook [30]:

$$
\begin{aligned}
\rho\left(E_{k}\right) & =Z^{-1}(\beta, V, N) \exp \left(-\beta E_{k}\right) \rightarrow \rho\left(\theta_{k}\right) \\
& =Z^{-1}\left(\beta_{\text {proj }}, V_{\text {proj }}, N_{\text {proj }}\right) \exp \left(-\beta_{\text {proj }} \theta_{k}\right),
\end{aligned}
$$

with the partition functions as

$$
\begin{aligned}
Z(\beta, V, N) & =\sum_{k} \exp \left(-\beta E_{k}\right) \rightarrow Z\left(\beta_{\text {proj }}, V_{\text {proj }}, N_{\text {proj }}\right) \\
& =\sum_{k} \exp \left(-\beta_{\text {proj }} \theta_{k}\right) \\
\beta & =\left(k_{B} T\right)^{-1} \rightarrow \beta_{\text {proj }}=\left(k_{B} T_{\text {proj }}\right)^{-1}
\end{aligned}
$$

where $\theta_{k}$ is an eigenvalue of $\Theta, \beta_{\text {proj }}$ is extended as a function of position and time. In fact, suppose the density distribution in quantum canonical system given by

$$
\rho\left(E_{k j}\right)=\frac{\exp \left(-\beta E_{k j}\right)}{\sum_{k j} \exp \left(-\beta E_{k j}\right)},
$$

which gives the density operator $\rho$ as

$$
\begin{aligned}
\rho & =\frac{1}{\operatorname{Tr} \exp (-\beta H)} \exp (-\beta H) \sum_{k j}\left|f_{k j}\right\rangle\left\langle f_{k j}\right| \\
& =\frac{\exp (-\beta H)}{\operatorname{Tr} \exp (-\beta H)} .
\end{aligned}
$$

Thus, using the similarity transformation $\Omega$ one can obtain a projected density operator $\rho_{\text {proj }}$ as

$$
\begin{aligned}
\rho_{\text {proj }} & =\Omega^{-1} \rho \Omega \\
& =\Omega^{-1} \frac{\exp (-\beta H)}{\operatorname{Tr} \exp (-\beta H)} \Omega=\frac{\exp \left(-\beta_{\text {proj }} \Theta\right)}{\operatorname{Tr} \exp \left(-\beta_{\text {proj }} \Theta\right)} .
\end{aligned}
$$

This gives a precise formula of the quantum canonical ensemble for a projected density operator $\rho_{\text {proj}}$. We consider this as generalizing the equilibrium quantum canonical ensembles formula to the non-equilibrium quantum canonical ensembles formula in the following sense: (i) if the similarity operator is unitary, then the new relations is just an effective representation of the old equilibrium quantum canonical ensembles relations because $\Theta$ or $H$ has the same spectral structure; (ii) if the similarity operator is non-unitary, then the new relations is an extension of the old relations that represents kind of non-equilibrium quantum canonical ensembles relations and reflects irreversibility of the system. The spectrum of $\Theta$ may appear to have complex spectral structure that is impossible to get from the original self-adjoint operator $H$ in the Hilbert space; and (iii) if the similarity operator can be deduced by some approximation, such as the Markovian/non-Markovian approximations, then the new relations can expose some non-equilibrium characteristics, which cannot be gained from the equilibrium quantum ensemble relations.

Thus it is obvious that the preceding construction of the density operator $\rho\left(\theta_{k}\right)$ can be extended to the classical statistical canonical ensemble by

$$
\rho\left(\theta_{k}\right)=Z^{-1}(\beta, V, N) \exp \left(-\beta_{\text {proj }} \theta_{k}\right)
$$

with

$$
Z(\beta, N, V)=\int \exp \left(-\beta_{\text {proj }} \Theta\right) \mathrm{d} \Gamma
$$

In the same way, the sub-dynamic grand canonical ensemble distribution can also be constructed by

$$
\rho\left(\theta_{k}\right)=Z^{-1}\left(\beta, \mu, V_{\text {proj }}\right) \exp \left[-\beta_{\text {proj }} \theta_{k}-\mu_{\text {proj }} N_{\text {proj }}\right],
$$

where the partition function is given by

$$
Z\left(\beta, \mu, V_{\text {proj }}\right)=\sum_{k} \int \exp \left[-\beta_{\text {proj }} \theta_{k}-\mu_{\text {proj }} N_{\text {proj }}\right] .
$$

Furthermore, the general canonical ensembles distribution can be written by

$$
\rho=Z^{-1} \exp \left[-\beta_{\text {proj }} \theta_{k}-\mu_{\text {proj }} N_{\text {proj }}-\sum_{k} \gamma_{k} \Gamma_{k}\right],
$$

where the thermodynamic meanings of the parameters $\gamma_{k}$ and $\Gamma_{k}$ can be fixed by the thermodynamic correspondence. Again, the physical meaning of $\Theta, \beta_{\text {proj }}, \mu_{\text {proj }}$ and $N_{\text {proj }}=$ $\Omega^{-1} N_{k} \Omega$ are also the "vacuum" of "dynamic part" of the corresponding parameters, which can be functionals of the coordinates of the system; when the $k$ th system in the ensemble tends to equilibrium, they tend to equilibrium $H_{k}, \beta$, $\mu$ and $N_{k}$, respectively. We emphasize again that in Balescue's book [30], the "dynamic part" means essence part of the (irreversible) evolution of the density distribution, and the "vacuum" means without correlation. His work and the Brussels-Austin school's later work seem to show that 
$\rho_{\text {proj }}$ plays an influential role in the (irreversible) evolution of the system by extending it to rigged Hilbert space or rigged Liouville space [31,32]. Using this means, the question is: can one build a corresponding relation between the equilibrium statistical ensemble formalism and non-equilibrium statistical ensemble formalism? The affirmation confirms that the original Hamiltonian of the system is related to the collision operator by the similarity transformation. Thus, the dynamic variables $Y$ are usually obtained by integrating over the non-equilibrium statistical distribution $\rho\left(\theta_{k}\right)$ which is given by the proposed non-equilibrium statistical ensemble relations (21) or (28) or from the solution of the SKE (26), $\langle Y\rangle=\operatorname{Tr}\left(Y \rho\left(\theta_{k}\right)\right)$. If the second order approximation of $\Theta$ corresponds to the master equation, the Boltzmann equation, the Pauli equation, or the FokkerPlanck equation, then $\operatorname{Tr}\left(Y \rho\left(\theta_{k}\right)\right)$ should deliver the expectation of corresponding physical value in the non-equilibrium ensembles. Eq. (25) can be a starting point to get non-equilibrium statistical ensembles formulations for irreversibility, as demonstration of application below.

\section{Kinetic equations and ensemble formulation}

For strong coupling, the kinetic equation is

$$
i \frac{\partial P_{n}}{\partial t}=\Theta P_{n}=H_{s} P_{n}+\lambda^{2} P_{n} H_{\mathrm{int}} G_{Q} H_{\mathrm{int}} P_{n},
$$

where $G_{Q}$ is a resolvent introduced as

$$
G_{Q}=\frac{1}{\theta-Q H Q}
$$

Consider the eigenvalue problem and the Born expansion series, one gets

$$
\begin{aligned}
\left(\Theta_{n}-H_{s}\right) P_{n} & =\lambda P_{n} H_{\mathrm{int}} P_{n}+\lambda^{2} P_{n} H_{\mathrm{int}} G_{Q} H_{\mathrm{int}} P_{n} \\
& =\lambda P_{n} H_{\mathrm{int}}\left(1+\lambda G_{Q}^{0} H_{\mathrm{int}}+\lambda^{2} G_{Q}^{0} H_{\mathrm{int}} G_{Q}^{0} H_{\mathrm{int}}+\cdots\right) P_{n} \\
& =\lambda P_{n} H_{\mathrm{int}} \frac{1}{1-\lambda G_{Q}^{0} H_{\mathrm{int}}} P_{n},
\end{aligned}
$$

where $G_{Q}$ is

$$
G_{Q}=\frac{1}{\theta-Q H_{s} Q}
$$

Since $\lambda>>1$, this may give $\lambda G_{Q}^{0} H_{\text {int }}>>1$ in the strong interaction; therefore, one obtains

$$
\left(\Theta_{n}-H_{s}\right) P_{n} \approx \lambda H_{\mathrm{int}} \frac{1}{-\lambda G_{Q}^{0} H_{\mathrm{int}}} P_{n},
$$

which gives

$$
\begin{aligned}
\left(\theta_{n}-E_{n}^{0}\right) P_{n} & \approx-P_{n} H_{\mathrm{int}}\left(\theta_{n}-Q_{n} H_{s} Q_{n}\right) H_{\mathrm{int}}^{-1} P_{n} \\
& =-\theta_{n} P_{n}+P_{n} H_{\mathrm{int}} Q_{n} H_{s} Q_{n} H_{\mathrm{int}}^{-1} P_{n} .
\end{aligned}
$$

Hence the eigenvalues are given by

$$
\begin{aligned}
\theta_{n} & \left.=\frac{1}{2}\left(E_{n}^{0}+\left\langle\varphi_{n}\right| H_{\mathrm{int}} Q_{n} H_{s} Q_{n} H_{\mathrm{int}}^{-1}\right)\left|\varphi_{n}\right\rangle\right) \\
& =\frac{1}{2}\left(E_{n}^{0}+\sum_{n^{\prime}} E_{n^{\prime}}^{0}\left\langle\varphi_{n}\left|H_{\mathrm{int}}\right| \varphi_{n^{\prime}}\right\rangle\left\langle\varphi_{n^{\prime}}\left|H_{\mathrm{int}}^{-1}\right| \varphi_{n}\right\rangle\right) .
\end{aligned}
$$

Therefore, the density operator for this system can be described by a spectral decomposition as

$$
\begin{aligned}
\rho_{\text {proj }} & =\frac{\exp (-\beta \Theta)}{\operatorname{Tr} \exp (-\beta \Theta)} \\
& \approx \frac{1}{\sum_{n} \mathrm{e}^{-\beta \theta_{n}}} \sum_{n} \mathrm{e}^{-\beta \theta_{n}}\left|\varphi_{n}\right\rangle\left\langle\varphi_{n}\right|,
\end{aligned}
$$

and an expectation for any observer $A$ in the quantum open system can be calculated by

$$
\begin{aligned}
\langle A\rangle & =\operatorname{Tr}(A \rho) \\
& \approx \frac{1}{\sum_{n} \exp \left(-\beta \theta_{n}\right)} \sum_{n} \exp \left(-\beta \theta_{n}\right)\left\langle\varphi_{n}|A| \varphi_{n}\right\rangle,
\end{aligned}
$$

where the interaction part, correlation part or dissipative part is

$$
\frac{1}{\sum_{n} \mathrm{e}^{-\beta \theta_{n}}} \sum_{n} \mathrm{e}^{-\frac{\beta}{2} \sum_{n^{\prime}} E_{n^{\prime}}^{0}\left\langle\varphi_{n}\left|H_{\text {int }}\right| \varphi_{n^{\prime}}\right\rangle\left\langle\varphi_{n^{\prime}}\left|H_{\text {int }}^{-1}\right| \varphi_{n}\right\rangle}\left|\varphi_{n}\right\rangle\left\langle\varphi_{n}\right|,
$$

and the partition function is

$$
Z=\frac{1}{\sum_{n} \mathrm{e}^{-\beta \theta_{n}}}
$$

\section{An application for the spin-Boson model}

The spin-boson model is an important model to deal with quantum system interactions with the environment [33] for which it is assumed that the environment consists of a set of harmonic oscillators. The spin-boson model can be far from equilibrium. For strong interaction, suppose a Hamiltonian for the spin-boson model is given by

$$
H=\in \sigma_{z}+\lambda \sigma_{\chi} \sum_{j} \alpha_{j}\left(b_{j}^{+}+b_{j}\right)+\sum_{j} \omega_{j} n_{j},
$$

where $\sigma_{z}=\left(\begin{array}{cc}1 & 0 \\ 0 & -1\end{array}\right)$, and $\sigma_{x}=\left(\begin{array}{ll}0 & 1 \\ 1 & 0\end{array}\right), \quad n_{j}=b_{j}^{+} b_{j}$ with $b_{j}^{+} b_{j}$ is a creation (annihilation) operator for the bosons (such as phonons or photons) within the environment, and $\lambda<<1$. The eigenvectors of the free Hamiltonian are

$$
\left|\phi_{1}\right\rangle=\left(\frac{1}{0}\right),\left|\phi_{2}\right\rangle=\left(\frac{0}{1}\right),\left|n_{k}\right\rangle,
$$


thus the expansion of $H$ with respect to the basis $\left(\left|\phi_{j} \otimes n_{k}\right\rangle,\left\langle n_{k} \otimes \phi_{j}\right|\right)$ is

$$
\begin{aligned}
& H=\sum_{j=1}^{2} \sum_{k}\left((-1)^{j+1} \in+\omega_{k} n_{k}\right)\left|\phi_{j} \otimes n_{k}\right\rangle\left\langle n_{k} \otimes \phi_{j}\right| \\
& +\lambda \sum_{j=1}^{2} \sum_{k} \alpha_{k}\left(\sqrt{n_{k}+1} \delta_{n_{k^{\prime}}, n_{k}+1}+\sqrt{n_{k}} \delta_{n_{k^{\prime}}, n_{k}+1}\right)\left|\phi_{j} \otimes n_{k}\right\rangle\left\langle n_{k^{\prime}} \otimes \phi_{j^{\prime}}\right|,
\end{aligned}
$$

where $j=j^{\prime}$ and $k=k^{\prime}$ are not present. The off-diagonal elements of the matrix for the Hamiltonian is

$$
\begin{aligned}
& \left\langle n_{k^{\prime}} \otimes \phi_{j^{\prime}}\left|\sigma_{\chi} \sum_{k} \alpha_{k}\left(b_{k}^{+}+b_{k}\right)\right| \phi_{j} \otimes n_{k}\right\rangle \\
& = \begin{cases}\sum_{k} \alpha_{k} \sqrt{n_{k}+1}, & \text { for } n_{k^{\prime}}=n_{k}+1, \\
\sum_{k} \alpha_{k} \sqrt{n_{k}}, & \text { for } n_{k^{\prime}}=n_{k}-1 .\end{cases}
\end{aligned}
$$

We introduce an eigen-projector as

$$
P_{j k}=\left|\phi_{j} \otimes n_{k}\right\rangle\left\langle n_{k} \otimes \phi_{j}\right|
$$

and

$$
Q_{j k}=1-P_{j k} .
$$

For strong interactions, i.e. $\lambda \sim \in$, or $\lambda \gg>1$ : then, the SKE becomes

$$
\begin{aligned}
i \frac{\partial \varphi_{\mathrm{proj}}}{\partial t}=\Theta \varphi_{\text {proj }} & =\left[H_{0}+\lambda H_{\mathrm{int}} C\right] \varphi_{\mathrm{proj}}, \quad \text { Then } \theta_{j k} \text { can be derived by } \\
\theta_{j k}= & \frac{E_{j k}^{0}+\left\langle n_{k} \otimes \phi_{j}\left|H_{\mathrm{int}} Q_{j k} H_{0} Q_{j k} H_{\mathrm{int}}^{-1}\right| \phi_{j} \otimes n_{k}\right\rangle}{2} \\
= & \frac{ \pm \in+\omega_{k} n_{k}+\sum_{k^{\prime} j^{\prime}}\left( \pm \in+\omega_{k^{\prime}} n_{k^{\prime}}\right)\left\langle n_{k} \otimes \phi_{j}\left|H_{\mathrm{int}}\right| \phi_{j^{\prime}} \otimes n_{k^{\prime}}\right\rangle\left\langle n_{k^{\prime}} \otimes \phi_{j^{\prime}}\left|H_{\mathrm{int}}^{-1}\right| \phi_{j} \otimes n_{k}\right\rangle}{2} \\
& \approx \frac{ \pm \in+\omega_{k} n_{k}+\frac{\left( \pm \in+\omega_{k^{\prime}}\left(n_{k}+1\right)\right)}{2}+\frac{\left( \pm \in+\omega_{k^{\prime \prime}}\left(n_{k}+1\right)\right)}{2}}{2}, \text { for } n_{k^{\prime}}=n_{k}+1, n_{k^{\prime \prime}}=n_{k}-1,
\end{aligned}
$$

which gives the above eigenvector $P_{j}$ for the $H_{0}$ to satisfy

$$
i \frac{\partial P_{j k}}{\partial t}=\Theta_{j k} P_{j k}=H_{0} P_{j k}+\lambda^{2} P_{j k} H_{\mathrm{int}} G_{Q} H_{\mathrm{int}} P_{j k},
$$

where $G_{Q}$ is defined as

$$
G_{Q}=\frac{1}{Z-Q_{j k} H Q_{j k}} .
$$

Using the above equation and considering the eigenvalue problem of $\Theta$ and the Born expansion of $G_{Q}$, one obtains

$$
\begin{aligned}
& \left(\Theta_{j k}-H_{0}\right) P_{j k} \\
= & \lambda P_{j k} H_{\mathrm{int}}\left(1+\lambda G_{Q}^{0} H_{\mathrm{int}}+\lambda^{2} G_{Q}^{0} H_{\mathrm{int}} G_{Q}^{0} H_{\mathrm{int}}+\cdots\right) P_{j k} \\
= & \lambda P_{j k} H_{\mathrm{int}} \frac{1}{1-\lambda G_{Q}^{0} H_{\mathrm{int}}} P_{j k},
\end{aligned}
$$

since $\lambda \sim \in$, or $\lambda \gg>1$, we can get

$$
\left(\Theta_{j k}-H_{0}\right) P_{j k}=\lambda P_{j k} H_{\mathrm{int}} \frac{1}{-\lambda G_{Q}^{0} H_{\mathrm{int}}} P_{j k},
$$

and

$$
\begin{aligned}
\left(\Theta_{j k}-H_{0}\right) P_{j k} & =-P_{j k} H_{\mathrm{int}}\left(\theta_{j k}-Q_{j k} H_{0} Q_{j k}\right) H_{\mathrm{int}}^{-1} P_{j k} \\
& =-P_{j k} \theta_{j k}+P_{j k} H_{\mathrm{int}} Q_{j k} H_{0} Q_{j k} H_{\mathrm{int}}^{-1} P_{j k} .
\end{aligned}
$$

which shows that $\theta_{j k}$ becomes independent of $H_{\text {int }}$ in the strong interaction approximation. This allows one to get a reduced density operator for the canonical system

$$
\begin{aligned}
\operatorname{Tr}_{B} \rho= & \operatorname{Tr}_{B}\left(\sum_{j, k}\left\langle\phi_{j k}|\rho| \phi_{j k}\right\rangle P_{j k}\right)=\frac{\operatorname{Tr}_{B} \exp (-\beta \Theta)}{\operatorname{Tr} \exp (-\beta \Theta)} \\
& \approx \frac{\sum_{j} \exp \left\{-\beta \sum_{k} \frac{ \pm \in+\omega_{k} n_{k}+\frac{\left( \pm \in+\omega_{k^{\prime}}\left(n_{k}+1\right)\right)}{2}+\frac{\left( \pm \in+\omega_{k^{\prime \prime}}\left(n_{k}+1\right)\right)}{2}}{2}\right\} P_{j}}{\sum_{j=1}^{2} \exp \left\{-\beta \sum_{k} \frac{ \pm \in+\omega_{k} n_{k}+\frac{\left( \pm \in+\omega_{k^{\prime}}\left(n_{k}+1\right)\right)}{2}+\frac{\left( \pm \in+\omega_{k^{\prime \prime}}\left(n_{k}+1\right)\right)}{2}}{2}\right\}} .
\end{aligned}
$$



sign.

If $j=1$, we select the "+" sign; if $j=2$, we select the "-"

Hence, the construction of the above system in the SKE subspace is quantum decoherence-free, which is useful for quantum computing. In fact, from eq. (54) one can see that the reduced density operator for the canonical system, $\operatorname{Tr}_{B \rho}$, is indeed independent of $H_{\text {int }}$ after the final approximation. $\theta_{j k}$ is only related to a part of the matrix of $H_{0}$, which means that the environment has no influence on the system $S$ and the system is decoherence-free in this case.

\section{Conclusions}

In conclusions, we have proposed subdynamic ensemble formalism based on the SSKE. The constructed procedure is to use a similarity transformation between the Gibbsian ensemble formalism based on the Liouville equation and the subdynamic ensemble formalism based on the SKE. Using this formalism, we study the spin-boson system with strong coupling, and obtain the reduced density operators for the canonical ensembles. We found that the reduced density operator is no longer related to $\lambda$ in the approximation, and show it possesses a kind of decoherence-free property. Moreover, a holographic concept for the universe through the SKE has also been proposed.

This work was supported by the National Natural Science Foundation of China (60874087).

1 Chapman S. Velocity-distribution in a non-uniform gas. Proc Roy Soc London A, 1916-17, 93: 1-20

2 Enskog D. Kinetische Theorie der Vorgäng in mässig verdünnten Gasen. Uppsala: Almqvist and Wiksells, 1917

3 Bogoliubov N N. Problems of dynamical theory in statistical physics. J Phys (USSR), 1946, 10: 256-265

4 Bogoliubov N N. Introduction to Quantum Statistical Mechanics. 2nd ed. Singapore: World Scientific Publishing 2010

5 Born M, Green H S. A general kinetic theory of liquids. I The molecular distribution function. Proc Roy Soc London A, 1946, 188: 10-18

6 Born M, Green H S. A general kinetic theory of liquids. III Dynamical process. Proc Roy Soc London A, 1947, 190: 455-474

7 Kirkwood J G. Statistical mechanical theory of transport processes. I. General theory. J Chem Phys, 1946, 14: 180-201

8 Kirkwood J G. Selected Topics in Statistical Mechanics. New York: Gordon and Breach, 1967

9 Yvon J. La Theorie Statistiques des Fluides et l'Equation d'Etat. Paris: Herman et Cie, 1935
10 Green M S. Markoff random processes and the statistical mechanics of time-dependent phenomena. II. Irreversible processes in fluids. J Chem Phys, 1954, 22: 398-413

11 Kubo R. Statistical-mechanical theory of irreversible processes. I. General theory and simple applications to magnetic and conduction problems. J Phys Soc Japan, 1957, 12: 570-586

12 Mori $\mathrm{H}$. Time-correlation functions in the statistical mechanics of transport processes. Phys Rev, 1958, 111: 694-706

13 Xing X S. On fundamental equation of statistical physics (II)*Nonequilibrium entropy and its evolution equation. Sci China Ser A-Math, 1998, 41: 411-421

14 Xing X S. New progress in the principle of nonequilibrium statistical physics. Chinese Sci Bull, 2001, 46: 448-454

15 Xing X S. On the fundamental equation of nonequilibrium statistical physics-Nonequilibrium entropy evolution equation and the formula for entropy production rate. Sci China Phys Mech Astron, 2010, 53: 2194-2215

16 Xing X S. Dynamic information theory and information description of dynamic systems. Sci China Phys Mech Astron, 2010, 53: 607627

17 Xing X S. Dynamic statistical information theory. Sci China Phys Mech Astron, 2006, 49: 1-37

18 Wang L N, Min J C. Thermodynamic analysis of adsorption process at a non-equilibrium steady state. Chinese Sci Bull, 2010, 55: 36193625

19 Zubarev D, Morozov V, Röpke G. Statistical Mechanics of Nonequilibrium Processes. Berlin: Akademie Verlag, 1996

20 Luzzi R, Vasconcellos Á R, Ramos J G. Predictive Statistical Mechanics-A Non-equilibrium Ensemble Formalism. Dordrecht: Kluwer Academic Publishers, 2002

21 Eu B C. Nonequilibrium Statistical Mechanics (Ensemble Method). Dordrecht, Boston, London: Kluwer Academic Publishers, 1998

22 Antoniou I, Tasaki S. Generalized spectral decompositions of mixing dynamical systems. J Quant Chem, 1993, 46: 425-474

23 Petrosky T, Prigogine I. Poincare resonances and the limits of trajectory dynamics. Physica A, 1991, 175: 146-209

24 Antoniou I, Melnikov Y, Bi Q. Master equation for a quantum system driven by a strong periodic field in the quasienergy representation. Physica A, 1997, 246: 97-114

25 Bi Q, Ruda H E, Zhang M S, et al. Kinetic equation, non-perturbative approach and decoherence free subspace for quantum open system. Physica A, 2003, 322: 345-358

26 Bi Q, Ruda H E, Zhou Z D. Dynamical equations of quantum information and Gaussian channel. Physica A, 2006, 363: 198-210

27 Prigogine I. From Being to Becoming. New York: Freeman, 1980

28 Nakajima S. On quantum theory of transport phenomena. Prog Theor Phys, 1958, 20: 948-959

29 Zwanzig R. Ensemble method in the theory of irreversibility. J Chem Phys, 1960, 33: 1338-1341

30 Balescu R. Equilibrium and Non-equilibrium Statistical Mechanics. New York: Wiley, 1975

31 Bi Q, Ruda H E, Zeng X H, et al. Extended space for quantum cryptography using mixed states. Physica A, 2003, 320: 357-370

32 Antoniou I, Prigogine I. Intrinsic irreversibility and integrability of dynamics. Physica A, 1993, 192: 443-464

33 Bi Q, Ruda H E, Zhan M S. Two-qubit quantum computing in a projected subspace. Phys Rev A, 2002, 65: 042325

Open Access This article is distributed under the terms of the Creative Commons Attribution License which permits any use, distribution, and reproduction in any medium, provided the original author(s) and source are credited. 